\section{Culture and insight revisited}

\author{
B. SARAVANAN, K. S. JACOB, M. PRINCE, D. BHUGRA and A. S. DAVID
}

Insight in psychosis is a complex and controversial phenomenon (David, 1990). A criticism of the concept is that it fails to take into account cultural idioms and is 'Eurocentric'.

\section{CONCEPTS AND CONTROVERSIES}

Transcultural studies of schizophrenia carried out under the auspices of the World Health Organization found that 'lack of insight' was an almost invariable feature of acute and chronic schizophrenia, regardless of setting (Wilson et al, 1986). The categorical or unidimensional view of insight has given way to more nuanced and multi-dimensional perspectives (Amador \& David, 1998). In the past decade, instruments have been devised to assess and quantify insight and important associations with psychopathology, social functioning and prognosis. These studies are largely from Western countries and problems regarding the cross-cultural validity have not been adequately discussed. Some authors (e.g. Perkins \& Moodley, 1993; Johnson \& Orrell, 1995; BeckSander, 1998) question this Western conceptualisation of insight and argue that it is relative and involves a comparative judgement (but see David, 1998). Our starting point is the biopsychosocial perspective from which we view insight as a culturally mediated human property that has survival value. However, there are several facets to this, each of which will be discussed below.

\section{Poor insight as a defence mechanism}

Lack of insight is often seen as a defence against the potentially devastating realisation of a person's illness. It is thus an active (motivated) effort to cope with or adapt to distress. In its extreme form - denial - it is a type of self-deception that protects the individual from threats to the self and involves exaggerated perceptions of control and self-efficacy. Sociopsychological research suggests that such biases in cognitive appraisal are the 'norm' and not exclusive reactions to crises. The frequently reported finding that 'preserved' insight is related to depressive symptoms (Mintz et al, 2003) in patients with schizophrenia and inversely related to self-deception (Moore et al, 1999) may be interpreted as evidence that poor insight serves as a defensive function.

It might be argued that the mechanisms underlying the concept of insight as a defence lie on a continuum encompassing all experiences, whether 'normal' or 'pathological'. The problem is that, outside the psychoses, there is less consensus as to whether an individual does or does not have insight into any given aspect of his or her behaviour or mental life and even less as to whether or not it is 'genuine'.

\section{Poor insight as misattribution}

Lack of insight may be viewed as misattribution, a form of cognitive error based on lack of information, systematic biases or idiosyncratic beliefs. Misattribution rests on the assumption that there is a correct attribution for symptoms and experiences with respect to some goal. This notion of correctness brings up the question of whether insight is a value-laden concept that is likely to change with changing medical concepts of illness as well as social norms for illness behaviour. Advocates of the health-belief model argue that an alternative, allegedly incorrect construction of reality may make sense within the patient's local world of meaning and, hence, has to be interpreted as a divergent perspective rather than as a lack of insight (Hughes et al, 1997).

Work employing case vignettes from both Western and Eastern settings has shown, contrary to these assumptions, that patients with psychosis have a very similar model of mental illness (including the need for medical treatment) to that of mental health professionals (Chung et al, 1997), but this may be separate from their own illness awareness (e.g. McEvoy et al, 1993).

\section{Individual models of mental illness}

The concept of insight has been criticised for being 'simplistic' and 'restrictive' (Perkins \& Moodley, 1993; Beck-Sander, 1998). Individuals' perspectives, beliefs and values should be taken into consideration when we assess something as complex as insight. This can provide the clinician and researcher with a greater understanding of different models of illness, help-seeking and mental health service acceptability. Some sociological studies of labelling and stigmatisation suggest that diagnosis, in effect an imposed biomedical model, has costs in reduced self-esteem and lower social status for the afflicted individual (Link et al, 1987). However, alternative models also have costs: conceptualising lack of insight in terms of social deviance rather than disease may be allied to the rejection of psychotropic medications and other effective therapies. Furthermore, although acceptance of the mental illness label may increase perceived stigma, this is not necessarily so (Warner et al, 1989). Explicit acknowledgement of mental illness is intimately bound up with a sense of loss; nevertheless, promotion of the medical model may reduce guilt and therapeutic nihilism and need not lead to lack of autonomy. This balance of costs and benefits will be tilted according to the person's social and cultural milieu and will be understood only in this context.

\section{Insight as a sociocultural process}

Conceptions of mental illness and its treatment often stem from normative social and cultural constructions. People can have various culturally shaped frameworks to explain their illnesses, all possibly valid (Kleinman, 1980). Socially oriented authors contend that technical definitions of insight are Eurocentric and that the metaphor of insight is profoundly shaped by cultural beliefs and practices. Interestingly, the growing number of non-Western studies (Tharyan \& Saravanan, 2000; full reference list available from the authors upon request) that examined the components of insight support its cross-cultural validity and the local adaptability of the assessment instruments. Although there are considerable variations in the mean total scores 
across studies, some interesting similarities have emerged. The insight item with the most striking consistency was the ability to relabel psychotic symptoms as pathological. This taps meta-cognition and is evident when a person begins to talk about and reflect upon, say, 'the voices' as distinct from either natural or supernatural communications (David, 1990). This aspect of insight may be, at least in part, a form of neuropsychological deficit somewhat independent of cultural influences (Rossell et al, 2003). As an analogy, one would expect a lesion of the frontal lobes to disrupt self-awareness and other executive functions regardless of ethnicity and cultural setting.

There are other explanations for this apparent consistency. It could reflect, on the one hand, invariant properties of the phenomena in question (e.g. hallucinations, delusions), or, on the other, the Procrustean nature of Western categories of psychopathology. Culturally informed exploration of these issues is required.

\section{INSIGHT AND CULTURE}

\section{Multiple models}

Even the multi-dimensional framework for insight mentioned above fails to acknowledge that people with psychiatric disorders can hold multiple beliefs about their problem; indeed, they may be diverse and contradictory. Similarly there is no one-toone correspondence between beliefs and consequent actions. Help-seeking behaviours have a special place in our concept of insight - as a dimension in itself and as an external validator. However, clinical experience suggests that patients can simultaneously seek help (action) from different sources whose frameworks and treatments contradict each other. Hence, naturalistic ('Western') explanations (e.g. disease, abnormality, infection, degeneration) may coexist with personalistic ('Eastern') explanations (e.g. supernatural causation, sin and punishment, karma). Naturalistic explanations are internal whereas personalistic explanations are often external. However, such explanations often coexist in many cultures. For example, it is common for people in India simultaneously to seek help and treatments from practitioners of modern medicine and from traditional healers/shamans and, provided that each does not claim exclusivity, this may not lead to conflict (Joel et al, 2003). We hypothesise that such multiple models may be advantageous, 'buffering' notions of loss and stigma and preventing social disintegration.

Cultural concepts of mental disorder are closely related to insight. International research indicates that the symptomatology, help-seeking and course of schizophrenia, as well as other psychiatric disorders, are strongly influenced by cultural interpretations (Sartorius et al, 1987). The speculations on the underlying mechanisms for the better prognosis of schizophrenia in developing countries have direct implications for the cultural constructions of insight because of the interactions of self and culture. If the individual self is a culturally mediated interpretation, then we might expect that cultures act through self-awareness to shape the natural course of schizophrenia.

Insight signifies a variety of ways in which a person's mental life approximates to that of others - in terms of what constitutes an illness, what beliefs are abnormal and what medical advice it is reasonable to follow. A number of shared assumptions allow these aspects to be incorporated in the mental model that psychiatrists have of what constitutes insight. This takes into account other clinical features, including history, course, culture, etc. In its own way, this is reliable and may even be valid. Hence, if a person could acknowledge some kind of non-visible change in his or her body or mind that affects the ability to function socially, and if he or she feels the need for restitution, then, irrespective of the attribution and the pathways of care that the person seeks, we could call this the presence of 'insight'.

\section{CONCLUSIONS AND FUTURE DIRECTIONS}

Insight is not only at the interface of biological and psychosocial explanations in psychiatry but also at the interface of globalisation and related cultural transitions. Globalisation and colonisation in various guises introduce new social effects and spread biomedical systems of thought, including causal explanations. Given these changes, how does a person find his or her way through this maze of differing opinions? And how do we know what to recommend when trying to improve the mental health of a diverse but increasingly interconnected world? Clearly there is a need for multi-disciplinary effort, including sociologists and anthropologists interested in insight research. In addition, future studies on insight should focus on the cross-cultural validity, reliability and methodological issues related to insight assessments. This must be complemented by open-ended enquiry to capture the complexity of representations and local political dimensions relevant to mental health and illness.

\section{DECLARATION OF INTEREST}

None.

\section{ACKNOWLEDGEMENTS}

B.S. is supported by a grant from the Wellcome Trust. The authors thank Dr C. David Goldberg for comments on an earlier draft.

\section{REFERENCES}

Amador, X. F. \& David, A. S. (1998) Insight and Psychosis. New York: Oxford University Press.

Beck-Sander, A. (1998) Is insight into psychosis meaningful? Journal of Mental Health, 7, 25-34.

Chung, K. F., Chen, E.Y., Lam, L. C., et al (1997) How are psychotic symptoms perceived? A comparison between patients, relatives and the general public. Australian \& New Zealand Journal of Psychiatry, 31, 756-761.

David, A. S. (1990) Insight and psychosis. British Journal of Psychiatry, I56, 798-808.

David, A. S. (1998) Commentary on: "Is insight into psychosis meaningful?' Journal of Mental Health, $\mathbf{7}$, 579-583.

Hughes, I., Hill, B. \& Budd, R. (1997) Compliance with antipsychotic medication: from theory to practice. Journal of Mental Health, 6, 473-489.

Joel, D., Sathyaseelan, M., Jayakaran, R., et al (2003) Explanatory models of psychosis among community health workers in South India. Acta Psychiatrica Scandinavica, 108, 66-69.

Johnson, S. \& Orrell, M. (1995) Insight and psychosis a social perspective. Psychological Medicine, 25, 515-520.

Kleinman, A. (1980) Patients and Healers in the Context of Culture. Berkeley, CA: University of California Press.

Link, B., Cullen, F. T., Frank, J., et al (1987) The social rejection of former mental patients: Understanding why labels matter. American Journal of Sociology, 92 , $|46|-1500$.

McEvoy, J. P., Schooler, N. J., Friedman, E., et al (1993) Use of psychopharmacology vignettes by patients with schizophrenia or schizoaffective disorder and by mental health professionals to judge patients' insight. American Journal of Psychiatry, 150, 1649-1653. 
Mintz, A. R., Dobson, K. S. \& Romney, D. M. (2003) Insight in schizophrenia: a meta-analysis. Schizophrenia Research, 6I, 75-88.

Moore, O., Cassidy, E., Carr, A., et al (1999) Unawareness of illness and its relationship with depression and self-deception in schizophrenia. European Psychiatry, 14, 264-269.

Perkins, R. \& Moodley, P. (1993) The arrogance of insight? Psychiatric Bulletin, 17, 233-234.

Rossell, S. L., Coakes, J., Shapeleske, J., et al (2003) Insight: its relationship with cognitive function, brain volume and symptoms in schizophrenia. Psychological Medicine, 33, $111-119$.

Sartorius, N., Jablensky, A., Ernberg, G., et al (1987) Course of schizophrenia in different cultures: Some results of a WHO international 5 year followup study. In Search for the Cause of Schizophrenia (eds H. Hafner,W. F. Gattaz \& W. Janzarik), pp. I07-II3. Berlin: Springer.

B. SARAVANAN, MD, K. S. JACOB, PhD, MD, Department of Psychiatry, Christian Medical College, Vellore, India; M. PRINCE, MRCPsych, D. BHUGRA, FRCPsych, A. S. DAVID, MD, FRCPsych, Institute of Psychiatry, De Crespigny Park, London, UK

Correspondence: Professor Anthony David, Section of Cognitive Neuropsychiatry, Institute of Psychiatry and GKT School of Medicine, Denmark Hill, London SE5 8AF, UK. Tel: 02078480138 ; fax 0207848 0572; e-mail: a.david@iop.kcl.ac.uk

(First received 2I February 2003, final revision 4 June 2003, accepted 3 July 2003)

Tharyan, A. \& Saravanan, B. (2000) lnsight and psychopathology in schizophrenia. Indian Journal of Psychiatry, 42, 421-426.

Warner, R., Taylor, D., Powers, M., et al (1989)

Acceptance of the mental illness label by psychotic patients: Effects on functioning. American Journal of Orthopsychiatry, 59, 398-409.

Wilson, W. H., Ban, T. A. \& Guys, W. (1986) Flexible system criteria in chronic schizophrenia. Comprehensive Psychiatry, 27, 259-265. 